\title{
Clincial Response of Central Nervous System Melanoma to Anti-PD1 Therapy in 2 Melanoma Patients
}

\section{Isabella C Glitza ${ }^{1 *}$ and Amanda D Bucheit ${ }^{2}$}

\author{
${ }^{1}$ Melanoma Medical Oncology, University of Texas MD Anderson Cancer Center, Houston, USA
}

${ }^{2}$ The START Center for Cancer Care, San Antonio, USA

\section{Introduction}

The development of Central Nervous System (CNS) disease, especially Leptomeningeal Disease (LMD), is a devastating complication in patients with metastatic melanoma. For LMD, life expectancy is typically measured in weeks, and current guidelines only recommend palliative radiotherapy or best supportive care [1]. Whole Brain Radiation (WBRT) is used in the management of CNS disease, but has limited impact on overall survival, and morbidity from its side effects should be considered $[2,3]$. For LMD, intrathecal immunotherapy with IL-2 has shown some promising results; however, this therapy is extremely toxic and should only be given to a select patient population [4]. While there are reports of CNS response to anti-PD1 treatment, little is known about the efficacy of anti-PD1 therapy in the treatment of LMD, though studies are currently ongoing (NCT02939300, NCT00338377) [5-7]. Here we report two cases of CNS melanoma patients, one with dural metastasis and a second with LMD, both achieving clinical benefit from treatment with anti-PD1 agents.

\section{Case Reports}

The first patient is a 76-year-old man who presented in June 2015 with aphasia and right hemiparesis. Imaging revealed a $3.5 \times 3 \times 1.3 \mathrm{~cm}$ left parietal mass. The patient underwent a craniotomy and pathology confirmed BRAF V600E mutant melanoma. He did not have a previous diagnosis of a primary melanoma. Subsequent PET imaging in August showed no evidence of systemic disease. Surveillance brain MRI in November showed several new nodular areas of enhancement along the dura and inter hemispheric falx, with CT of the body again not demonstrating other sites of disease. He was treated with whole brain radiation in December of 2015 (37.5 Gy, 15 fractions). In late January 2016 an MRI was done demonstrating mild progression of the anterior falcine dural metastatic disease. Given the progression was not significant and the patient was asymptomatic a watchful waiting approach was planned with a close follow-up MRI. Repeat MRI in March showed stable changes, thus surveillance continued.

Surveillance brain MRI in August 2016 revealed multiple new dural based enhancing metastatic nodules and enhancing nodularity in the posterior surgical bed, as well as worsening disease in the interhemispheric falx (Figure 1A). CT of the chest revealed a new lung nodule, suspicious for metastatic disease. Neurological exam was unremarkable, with an ECOG Performance Status (PS) of 0 . Cerebrospinal fluid collection was not performed. Treatment with nivolumab was initiated at $240 \mathrm{mg}$ IV every two weeks. The patient tolerated the treatment well with no noticeable toxicity. Brain MRI after $6 \mathrm{cy}$ cles showed a partial response and repeat MRI after 13 cycles showed a complete radiographic response (Figure 1B). At time of this report, he has received 14 doses of nivolumab without any toxicity and no neurological symptoms. His ECOG PS remained 0.

The second patient is 61-year-old female, who presented with sudden onset of expressive aphasia in November 2015. CNS imaging revealed a $1.9 \times 1.3 \times 1.1 \mathrm{~cm}$ mass protruding anteriorly into the third ventricle lead-

*Corresponding author: Isabella C Glitza, University of Texas MD Anderson Cancer Center, Melanoma Medical Oncology, 1515 Holcombe Boulevard, Houston, USA, Tel: 713-7922921, Fax: 713-745-1046, E-mail: ICGlitza@mdanderson.org

Received: February 10, 2017: Accepted: May 04, 2017: Published online: May 06, 2017

Citation: Glitza IC, Bucheit AD (2017) Clincial Response of Central Nervous System Melanoma to Anti-PD1 Therapy in 2 Melanoma Patients. Arch Immunol 1(1):1-3

Copyright: (c) 2017 Glitza IC, et al. This is an open-access article distributed under the terms of the Creative Commons Attribution License, which permits unrestricted use, distribution, and reproduction in any medium, provided the original author and source are credited. 


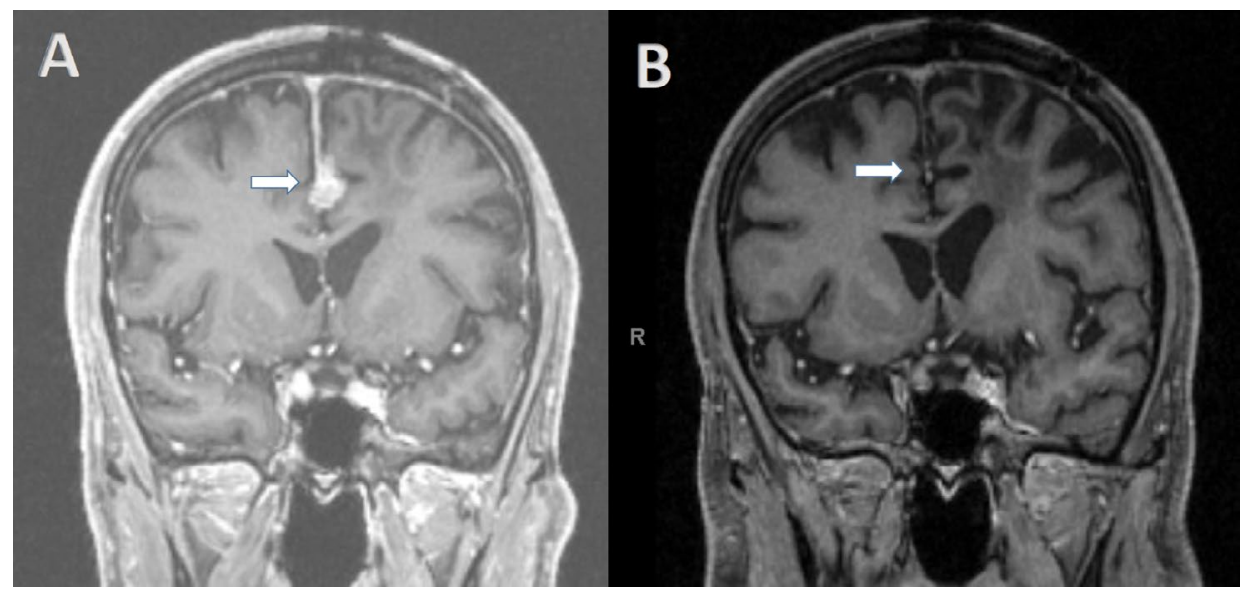

Figure 1: A) Baseline nodular enhancement (arrow) before; B) After treatment with nivolumab.

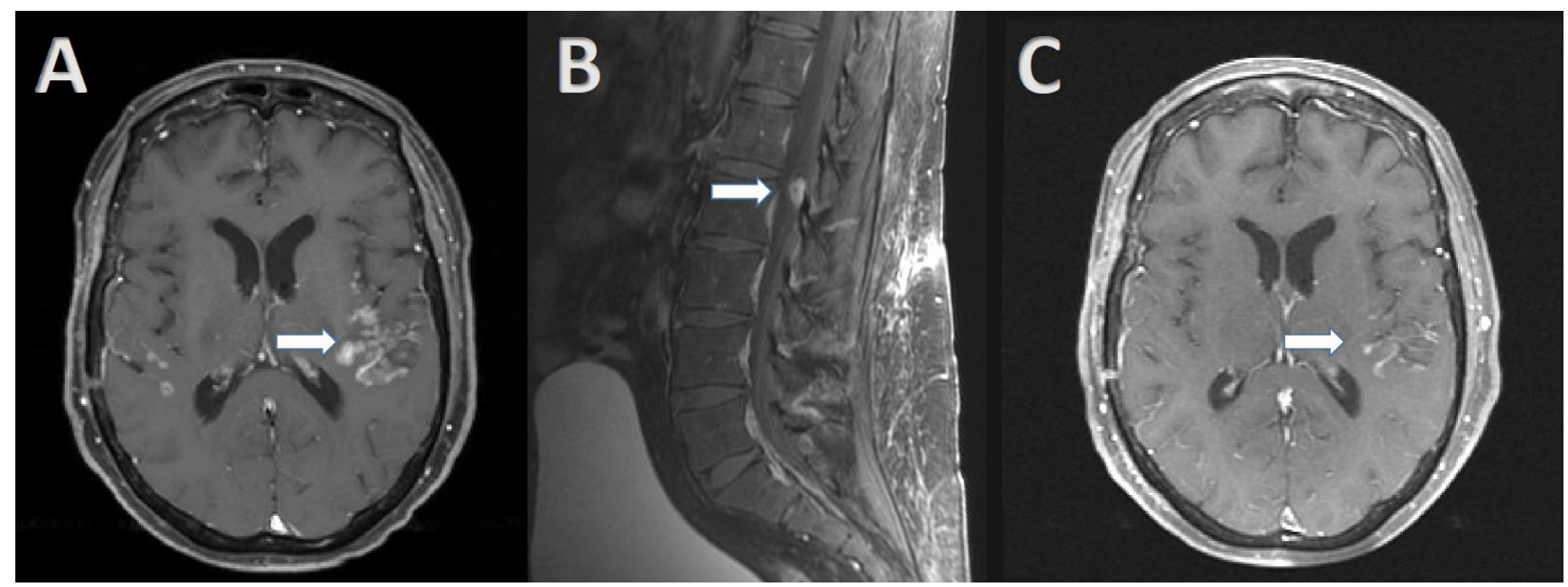

Figure 2: A) Baseline diffuse leptomeningeal enhancement (arrow) before; B) After treatment with pembrolizumab; C) Epidural involvement at $\mathrm{L} 1$ and $\mathrm{L} 2$ remained stable through treatment and did not require radiation.

ing to obstructive hydrocephalus as well as widespread leptomeningeal disease (Figure 2A). Her past medical history was unremarkable, and she had no history of primary melanoma. She underwent craniotomy and CSF analysis (positive cytology), and pathology confirmed melanoma. 50-Gene Somatic Mutation Analysis did not reveal any targetable mutation. Staging scans did not reveal any sites of systemic disease; however MRI spine showed epidural involvement at L1 and L2, with extension into neural foramina (Figure $2 \mathrm{~B}$ ). Whole brain radiation was initiated December 2015 (10 fractions, 30 Gy total). Treatment was complicated by recurrence of aphasia, requiring dexamethasone $(4 \mathrm{mg}$ three times a day), however, she was able to be weaned of the corticosteroids within 2 weeks. ECOG PS was 2, however, no residual neurological sequelae were noted. In February 2016, pembrolizumab ( $2 \mathrm{mg} / \mathrm{kg}$ ) was started. Follow up MRI brain after 3 cycles of pembrolizumab showed partial response (Figure 2B), and clinically she improved to ECOG PS 1. Two months later after a total of 6 cycles of pembrolizumab MRI brain showed continued response, with an ECOG of 0. In December of 2016, MRI brain revealed worsening LMD, but her PS and neurological exam remained unchanged. She was initiated on ipilimumab $(3 \mathrm{mg} / \mathrm{kg})$ and nivolumab $(1 \mathrm{mg} / \mathrm{kg})$, and both clinical and radiological exams were stable in March 2017. Spinal MRI remained unchanged. She remains on nivolumab $(3 \mathrm{mg} / \mathrm{kg})$ every 2 weeks without any toxicities.

\section{Discussion}

CNS disease, particularly LMD, represents a significant challenge in the treatment of melanoma patients, not only due to limited treatment options, but also due to significant morbidity and poor survival associated with this diagnosis. Recent studies have shown that immunotherapy can lead to responses in the brain, but LMD patients were excluded from these trials [5,6]. Our first patient not only obtained a complete CNS response, but also has not recurred at the time of this report. Our sec- 
ond patient also derived benefit from anti-PD1 therapy, and to our knowledge is the first melanoma LMD patient to derive benefit from anti-PD1 antibodies reported in the literature. Both patients received WBRT, however, the first patient did not receive the immunotherapy until 8 months after radiation, suggesting that the observed response is based on the efficacy of the anti-PD1 antibody alone. The second patient received the checkpoint inhibitor very shortly after WBRT, and a synergistic effect of radiotherapy cannot be entirely excluded. However, WBRT has not shown to improve the overall survival in melanoma patients with LMD, and is typically reserved for palliation of symptoms. Even more importantly, our LMD patient is alive and asymptomatic for over 16 months after her diagnosis of LMD.

While our experience with these two patients is encouraging, the results of ongoing trials are anxiously awaited, and will shed further light into the efficacy of anti-PD1 agents in the treatment of CNS disease, particularly LMD.

\section{References}

1. Cohen JV, Tawbi H, Margolin KA, et al. (2016) Melanoma Central Nervous System Metastases: Current Approaches,
Challenges, and Opportunities. Pigment Cell Melanoma Res 29: 627-642.

2. Hyun JW, Jeong IH, Joung $A$, et al. (2016) Leptomeningeal metastasis: Clinical experience of 519 cases. Eur J Cancer 56: 107-114.

3. Brown PD, Pugh S, Laack NN, et al. (2013) Memantine for the prevention of cognitive dysfunction in patients receiving whole-brain radiotherapy: a randomized, double-blind, placebo-controlled trial. Neuro Oncol 15: 1429-1437.

4. Papadopoulos NE, Gerber DL, Eton O, et al. (2002) The role of intrathecal use of interleukin-2 in the treatment of leptomeningeal disease in patients with melanoma. ASCO, Poster Discussion Session, Central Nervous System Tumors. Proc Am Soc Clin Oncol 21.

5. Goldberg SB, Gettinger SN, Mahajan A, et al. (2016) Pembrolizumab for patients with melanoma or non-small-cell lung cancer and untreated brain metastases: early analysis of a non-randomised, open-label, phase 2 trial. Lancet Oncol 17: 976-983.

6. Margolin K, Ernstoff MS, Hamid O, et al. (2012) Ipilimum$\mathrm{ab}$ in patients with melanoma and brain metastases: an open-label, phase 2 trial. Lancet Oncol 13: 459-465.

7. Dudnik E, Yust-Katz S, Nechushtan H, et al. (2016) Intracranial response to nivolumab in NSCLC patients with untreated or progressing CNS metastases. Lung Cancer 98: 114-117. 